\title{
Toroidal Effects on ICRF Heating and Current Drive
}

\author{
J. Carlsson, T. Hellsten ${ }^{\dagger}$, and J. Hedin ${ }^{\dagger}$ \\ Oak Ridge National Laboratory, Oak Ridge, Tennessee 37831-8071 \\ † Alfvén Laboratory, Royal Institute of Technology, S-100 44 Stockholm, Sweden
}

\begin{abstract}
Numerical studies, performed with the Monte-Carlo code FIDO [1], of the evolution of the resonant-ion distribution function in the presence of ICRH in toroidal geometry are presented. In particular it is pointed out how the absorption of toroidal momentum from a wave field with finite parallel wave numbers causes spatial drift and diffusion, which together with the finite orbit widths of the tail ions is shown to have a large effect on the temperature profile of the resonant ion species and also to cause losses of high-energy ions to the wall [2].

Furthermore, it is found that the finite orbit width and the inward drift occuring for negative parallel wave numbers [3] each give rise to a new mechanism of minority-ion cyclotron current drive as compared to earlier models where the drift orbits of the resonant ions are confined to the magnetic flux surfaces. For high levels of coupled power these new mechanisms are found to be the dominating ones $[4,5]$.
\end{abstract}

\section{INTRODUCTION}

For the fast wave the parallel component of the electric field $\mathbf{E}$ is zero. The equation of motion for an ion (charge $e Z$ and mass $m$ ) in a static magnetic field $\mathbf{B}_{\mathbf{0}}=\hat{\mathbf{z}} \mathbf{B}_{\mathbf{0}}$ is then $m \dot{\mathbf{v}}=e Z\left[\mathbf{E}+\mathbf{v} \times\left(\mathbf{B}_{\mathbf{0}}+\mathbf{k} \times \mathbf{E} / \omega\right)\right]$. If we take the scalar product of the equation of motion with $\mathbf{v}$ and $\mathbf{v}_{\perp}$, respectively, we find,

$$
\dot{W}_{\perp}=\left(1-\frac{k_{\|} v_{\|}}{\omega}\right) \dot{W}, \dot{W}_{\|}=\frac{k_{\|} v_{\|}}{\omega} \dot{W},
$$

i.e. for finite $k_{\|}$there is always some absorption of parallel energy $W_{\|}$(the work performed by the parallel component of the Lorentz force) in addition to the dominating absorption of perpendicular energy $W_{\perp}$. Another, perhaps more familiar, formulation is to say that in $\left(v_{\|}, v_{\perp}\right)$-space the quasilinear diffusion is always such that the ion moves along semi-circles centered at $\left(v_{\|}, v_{\perp}\right)=\left(\omega / k_{\|}, 0\right)$ see fig. 1 below.

In an axisymmetric torus the canonical toroidal momentum $P_{\phi}=m R v_{\|} B_{\phi} / B+$ $e Z \tilde{\psi}$ is a constant of the motion. $P_{\phi}$ couples the velocity space $\left(v_{\|}\right)$to the configuration space $(\tilde{\psi})$ causing drift motion across the flux surfaces. A perturbation 


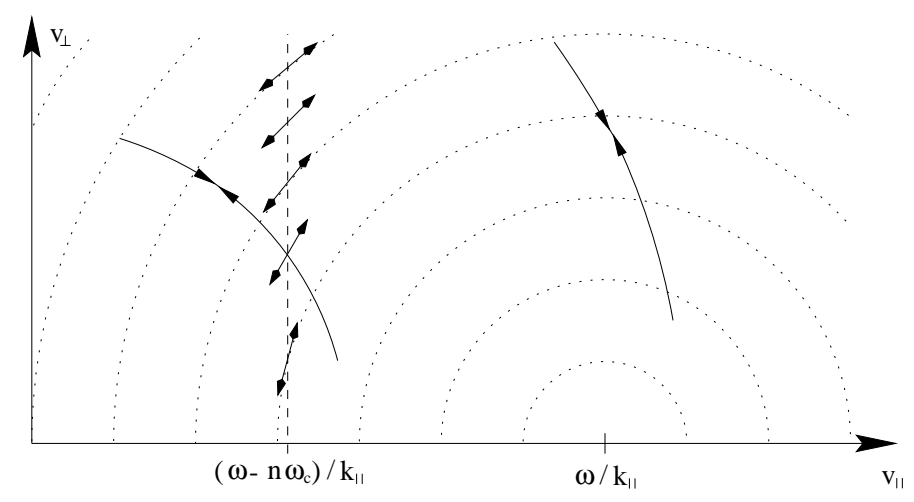

FIGURE 1. Cyclotron absorption in velocity space.

in velocity space is thus accompanied by a perturbation in configuration space. Coulomb collisions give rise to the neoclassical diffusion and the quasilinear drift and diffusion in velocity space leads to spatial drift and diffusion. The change in $P_{\phi}$ from wave-particle interaction is related to the change in energy through,

$$
\Delta P_{\phi}=\frac{R k_{\|}}{\omega} \Delta W
$$

Since the expectation value of $\Delta W$ is positive this means that for positive $k_{\|}$there will be an outward drift of resonant ions and an inward drift for negative $k_{\|}$.

\section{THE FIDO CODE}

The Monte-Carlo code FIDO [1] takes the wide non-standard drift orbits and the wave-induced spatial drift and diffusion into account by solving the quasilinear Fokker-Planck equation in axisymmetric toroidal geometry, retaining the $\partial / \partial v_{\|^{-}}$ term in the quasilinear operator.

The drift (deterministic, $\mathcal{O}(\Delta t)$ ) and diffusive (stochastic, $\mathcal{O}\left((\Delta t)^{1 / 2}\right)$ ) MonteCarlo kicks are averaged over unperturbed drift orbits. The quasilinear kicks are orbit-averaged analytically and the Coulomb kicks numerically. After orbit averaging the physics is modeled as drift and diffusion in a 3-D COM space making it possible to tabulate and interpolate the Monte-Carlo kicks in order to reduce execution times. Orbit averaging also removes the bounce period as a limit on the time step, making the shortest collisional (typically pitch-angle scattering) time scale the limit instead. This allows increasing the time step from the $\mu s$ to the $m s$ range.

Keeping the absorbed power constant in the code couples all the test particles but FIDO nevertheless scales well up to at least 32 nodes. 


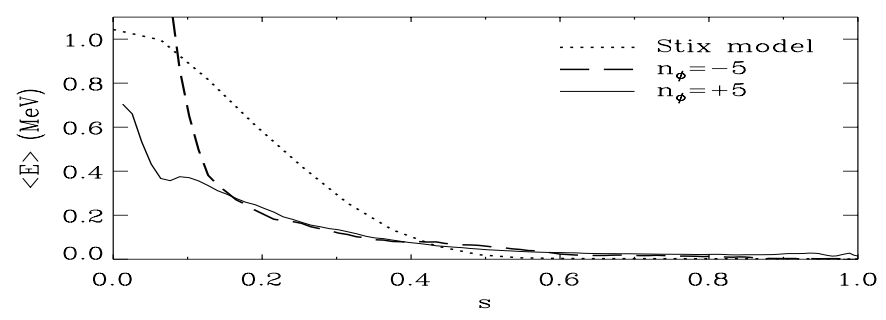

FIGURE 2. Profiles of the mean resonant-ion energy.

\section{MINORITY ION CYCLOTRON HEATING}

Using a neutral particle analyzer the high-energy tail formation up to one or a few $\mathrm{MeV}$ shows little dependence on the $k_{\|}$-spectrum in JET. Other integrated quantities, such as the total fast-ion energy or fusion yield, also indicate that the finite orbit width and wave-induced transport are of minor importance. However, a more careful, radially resolved investigation, shows that the tail formation locally, on a specific flux surface, does depend strongly on the $k_{\|}$-spectrum, see fig. 2 above [2].

The conclusion is that in a large tokamak the total energy absorption does not depend sensitively on $k_{\|}$but the radial distribution, out to and including the limiter (orbit losses), of the energy does [2].

Since the distribution function of the resonant species is strongly dependent

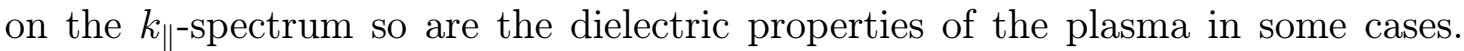
The importance of self-consistently solving the quasilinear Fokker-Planck and wave equations has been demonstrated for a FWCD scenario [6].

\section{MINORITY ION CURRENT DRIVE}

For a large tokamak with levels of coupled power in the MW range the model sketched above introduce two major new effects on the minority current drive as compared to models relying on the zero orbit-width approximation: the waveinduced spatial drift and the fact that the trapped tail ions, which often dominate the power absorption, contribute to the current. The trapped current is always bidirectional, reversing a bit outside the resonant surface, locally flattening the ohmic current. In some cases the direct absorption of parallel momentum from the wave also affect the driven current profile $[4,5]$.

Convergence with the Fisch model is found only in the limit of very low power densities (a few $\mathrm{kW} / \mathrm{m}^{3}$ ) and artificially high plasma current . At more typical power levels the asymmetric heating of the Fisch model was found to be of lesser importance than the two major effects mentioned above [5].

For positive $k_{\|}$the trapped tail ions dominate the power absorption. Due to the strong $v_{\perp}$-diffusion detrapping of tail ions, in combination with the asymmetric 


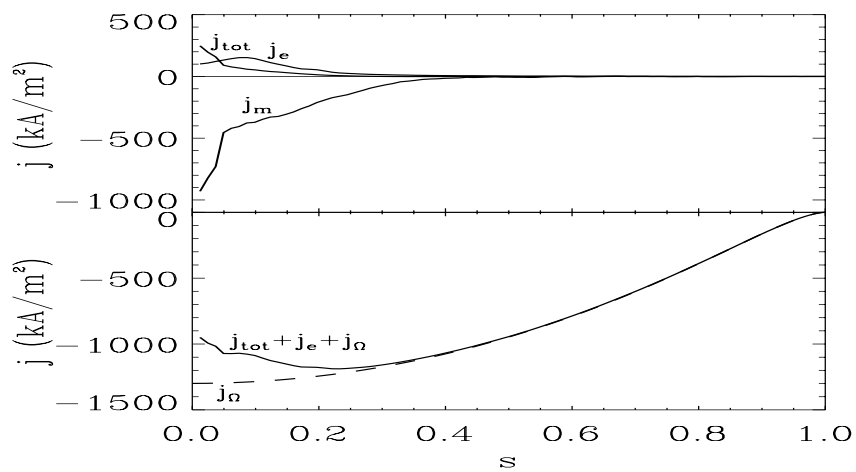

FIGURE 3. Driven current in an ITER plasma with on-axis resonance, $k_{\|} \approx-5.4 \mathrm{~m}^{-1}, 50 \mathrm{MW}$ of coupled power ( $42 \%$ to $\alpha$ and $56 \%$ to $e$ ). $j_{m}$ is the minority current, $j_{\text {tot }}$ is the minority current minus the back current from majority ions and electrons, $j_{e}$ is the electron current driven by FWCD and $j_{\Omega}$ is the ohmic current.

heating and absorption of parallel momentum, results in roughly equal contributions to the driven current from trapped and passing ions [5].

For negative $k_{\|}$the wave-induced spatial drift pulls the tail ions inward. If the resonance is close to the magnetic axis this drift can lead to asymmetric detrapping, creating a population of fast, counter-passing ions around the magnetic axis. At high levels of coupled power (5-10 MW) this mechanism totally dominates, resulting in a minority current which sharply peaks the ohmic current [5].

If $Z>Z_{\text {eff }}$ the back current is larger than the minority current. This is taken advantage of in an ITER case where inward wave-induced drift of the resonant fusion-born $\alpha$ 's in combination with FWCD produces negative shear as shown in fig. 3 above [5].

\section{REFERENCES}

1. Carlsson J., Eriksson L.-G., and Hellsten T., Proceedings of the Joint VarennaLausanne Workshop "Theory of Fusion Plasmas", Bologna: Editrice Compositori, 1994, p. 351.

2. Carlsson J., Eriksson L.-G., and Hellsten T., Nucl. Fusion 37, 719 (1997).

3. Eriksson L.-G., Mantsinen M., Borba D., Fasoli A., Heeter R., Sharapov S., Start D. F. H., Carlsson J., Gondhalekar A., Hellsten T., and Korotkov A., Phys. Rev. Lett. 81, 1231 (1998).

4. Hellsten T., Carlsson J., and Eriksson L.-G., Phys. Rev. Lett. 74, 3612 (1995).

5. Carlsson J., Hellsten T., and Hedin J., Phys. Plasmas 5, 2885 (1998).

6. Hedin J., Hellsten T., and Carlsson J., Proceedings of the Joint Varenna-Lausanne Workshop "Theory of Fusion Plasmas", Bologna: Editrice Compositori, 1998, p. 467.

7. Carlsson J., "Ion cyclotron resonance heating and current drive in tokamaks", $\mathrm{PhD}$ thesis, Royal Institute of Technology, ISBN 91-7170-237-7, 1998. 